\title{
Carboxymethyl sago pulp/chitosan hydrogel as an immobilization medium for activated sludge for $p$-nitrophenol biodegradation
}

\author{
Si Ling Ng $\mathbb{1}^{1}{ }^{1}$ Kevin Jenn Ling Yong, ${ }^{2}$ Janarthanan Pushpamalar, ${ }^{2,3}$ Suat Peng Sam ${ }^{1}$ \\ ${ }^{1}$ School of Chemical Sciences, Universiti Sains Malaysia, 11800 USM, Penang, Malaysia \\ ${ }^{2}$ School of Science, Monash University Malaysia, Jalan Lagoon Selatan, Bandar Sunway, 47500 Subang Jaya, Selangor, Malaysia \\ ${ }^{3}$ Monash-Industry Palm Oil Education and Research Platform (MIPO), Monash University Malaysia, Jalan Lagoon Selatan, Bandar \\ Sunway, Jalan Lagoon Selatan, Bandar Sunway, 47500 Selangor Darul Ehsan, Selangor, Malaysia \\ Correspondence to: S. L. Ng (E-mail: slng@usm.my)
}

ABSTRACT: In this study, carboxymethyl sago pulp (CMSP) derived from sago waste was successfully crosslinked with ferric ions in the presence of chitosan forming a novel immobilization matrix for $p$-nitrophenol (PNP)-acclimated activated sludge. On the basis of the shortest biodegradation time of PNP, the optimized operational conditions of immobilization were found to be: $7 \mathrm{w} / \mathrm{v} \% \mathrm{CMSP}, 9 \mathrm{~g} \mathrm{~L}^{-1}$ of activated sludge, $0.1 \mathrm{M}$ ferric ion, and $15 \mathrm{~min}$ of crosslinking time. Observable inhibited PNP biodegradation was exhibited by the suspended activated sludge at the initial PNP concentration of $400 \mathrm{mg} \mathrm{L}^{-1}$. In contrast, complete mineralization was achieved by the CMSP/chitosan-immobilized activated sludge (CMSP/Ch-AS) beads. The results revealed the important role of CMSP/Ch hydrogel in protecting activated sludge from the toxicity of PNP. The CMSP/Ch-AS beads could be reused consecutively up to three and two cycles, respectively, for the biodegradation of PNP at 200 and $400 \mathrm{mg} \mathrm{L}^{-1}$, with the attainment of more than $99 \%$ of PNP removal at each cycle. $\odot 2019$ Wiley Periodicals, Inc. J. Appl. Polym. Sci. 2019, 136, 47531.

KEYWORDS: activated sludge; biodegradation; carboxymethyl sago pulp/chitosan hydrogel; immobilization; p-nitrophenol

Received 28 March 2018; accepted 9 December 2018

DOI: 10.1002/app.47531

\section{INTRODUCTION}

Wastewater containing phenolic compounds from industrial sectors such as pharmaceuticals and petrochemical industries is a worldwide concern as these phenolic compounds pose a health hazard to humans and the environment due to their toxicity, carcinogenicity, and high bioaccumulation rate. ${ }^{1}$ The phenolic compounds such as $p$-nitrophenol (PNP) have been listed as priority pollutants by United States environmental protection agency and the concentration of PNP in the natural water bodies is restricted to below $10 \mathrm{ng} \mathrm{L}^{-1}$. $^{2}$ Thus, it is essential to treat these wastewaters properly prior to discharge them into receiving water.

With regard to the removal of phenolic compounds, biological treatment method using activated sludge is usually of preference compared to conventional physicochemical treatment methods due to its relatively low cost with the attainment of complete mineralization. ${ }^{3,4}$ Nonetheless, the conventional activated sludge process experiences several drawbacks including large reactor volume, low biomass concentration, production of excess sludge and washout, substrate inhibition on microbial activities at high concentrations of toxic pollutants as well as difficulty in solid-liquid separation. ${ }^{3,5,6}$ These problems can be minimized by using the immobilization system in addition to achieving a more easily controlled and efficient operation.
Four main methods of immobilization, namely, adsorption, surface binding, entrapment, and encapsulation have been discussed by Dzionek et al. ${ }^{8}$ Among these methods, entrapments of microorganisms using polymeric materials are widely used in the biological treatment system. A feasible polymeric material used as entrapment medium should be nontoxic to microorganisms, possess porous structure to facilitate the diffusion of substrates and products but prevents the leaking of cells and exhibits high mechanical stability. Various natural and synthetic organic polymers such as $\kappa$-carrageenan, alginate, chitosan, poly(vinyl alcohol), poly(ethylene glycol), and polyacrylamide $e^{3,7,9-14}$ have been successfully used to immobilize microbes. However, information on the optimization of immobilization conditions is relatively limited.

In the present study, sago waste was used as the precursor to developing carboxymethyl sago pulp chitosan (CMSP/Ch) hydrogel beads and used as immobilization medium to entrap PNP-acclimated activated sludge for the biodegradation of PNP. Abundant of sago waste was produced as a by-product from sago flour production in Malaysia and the amount is increasing due to the high demand of sago flour. ${ }^{15}$ The sago waste was reported to be disposed into the river, and it would be a wastage of valuable raw material. ${ }^{16}$ Besides, the disposal of sago waste also results in environmental issues such as oxygen depletion caused by the degradation of sago waste. Therefore, 
utilization of the sago waste as the starting material in forming polymers is of interest as they are inexpensive and biocompatible. Sago pulp could be extracted from sago waste and be converted to anionic CMSP through etherification of the hydroxyl group. ${ }^{17}$ The CMSP could be crosslinked forming polymer network that is suitable as immobilization matrix. CMSP hydrogel beads have been exploited extensively as carriers in different drug delivery systems. ${ }^{17-20}$ In our previous study with regard to the application of CMSP in wastewater treatment, ${ }^{15}$ CMSP produced from sago waste has successfully immobilized powdered activated carbon (PAC) and the CMSP-PAC beads formed were used to remove methylene blue from aqueous solutions. Nonetheless, the investigation on the feasibility of using CMSP in immobilizing viable biomass is lacking.

During our attempt to immobilize the activated sludge into CMSP hydrogel beads, it was noted that the beads formed were not stable enough to be employed for a complete cycle of biodegradation process (data not shown). Therefore, in order to strengthen the structure of the hydrogel beads, chitosan (Ch) was added during the immobilization process. Chitosan, a cationic polysaccharide derived from chitin, is one of the widely used polymer in the immobilization due to its nontoxicity and biocompatibility properties. ${ }^{10}$ It was reported that the addition of chitosan resulted in the formation of a coating at the hydrogel bead surface and thus enhanced its mechanical strength. ${ }^{10,21}$

In view of the above observations, the objectives of this study are to: (1) develop CMSP/chitosan-immobilized activated sludge (CMSP/Ch-AS) beads used for PNP biodegradation, (2) determine the optimized operational conditions for the formation of CMSP/Ch-AS beads, and (3) access the reusability of CMSP/Ch-AS beads in degrading PNP.

\section{MATERIALS AND METHODS}

\section{Culturing of PNP-Acclimated Activated Sludge}

PNP-acclimated activated sludge was cultured using sequencing batch reactor (SBR) with the 24-h operation mode of FILL: REACT:SETTLE:DRAW:IDLE (cycle time ratio of 1:12:2:0.5:8.5). The seed of the activated sludge was collected from a local municipal sewage treatment plant at Batu Ferringhi, Penang, Malaysia and acclimated to base mix containing (in $\mathrm{mg} \mathrm{L}^{-1}$ ): bacto-peptone (188), sucrose (563), $\left(\mathrm{NH}_{4}\right)_{2} \mathrm{SO}_{4}(212), \mathrm{K}_{2} \mathrm{HPO}_{4}(180), \mathrm{KH}_{2} \mathrm{PO}_{4}$ (32), $\mathrm{MgSO}_{4}$ (49), $\mathrm{CaCl}_{2}$ (40), $\mathrm{FeCl}_{3}$ (18.8), and $\mathrm{NaHCO}_{3}$ (354). After the attainment of steady state, bacto-peptone and sucrose were gradually replaced with PNP as the sole carbon source. The final acclimation PNP concentration was $200 \mathrm{mg} \mathrm{L}^{-1}$. The sludge retention time was controlled at 20 days by drawing $250 \mathrm{~mL}$ of mixed liquor at the end of REACT period daily. Upon attaining a steady state, the PNP-acclimated activated sludge was used for the preparation of CMSP/Ch-AS beads.

\section{Optimization of Immobilization}

Preparation of CMSP. The CMSP was prepared from sago waste following the procedure described in Thenapakiam et al. ${ }^{18}$ In this study, the CMSP with an average degree of substitution (DS) of 0.8 was chosen due to its lower swelling capacity and thus a more stable gelled structure could be obtained. To produce CMSP with DS 0.8 , briefly, $\sim 20 \mathrm{~g}$ of sago waste was suspended in $640 \mathrm{~mL}$ of hot distilled water in a $1000-\mathrm{mL}$ Schott bottle. Subsequently, a total of $4 \mathrm{~mL}$ of glacial acetic acid and $6 \mathrm{~g}$ of sodium chlorite were added and the mixture was heated to $70{ }^{\circ} \mathrm{C}$ for $3 \mathrm{~h}$. The product was then filtered and washed with $2 \mathrm{~L}$ of cold distilled water. The white residue, which was the sago pulp, was then dried in an oven at $60{ }^{\circ} \mathrm{C}$.

During carboxymethylation process, a total of $5 \mathrm{~g}$ of sago pulp was added into $100 \mathrm{~mL}$ of isopropanol, followed by the gradual addition of $10 \mathrm{~mL}$ of $30 \mathrm{w} / \mathrm{v} \%$ sodium hydroxide and the mixture was agitated for an hour at $160 \mathrm{rpm}$. The agitation was continued at $45{ }^{\circ} \mathrm{C}$ for $3 \mathrm{~h}$ after the addition of $6.0 \mathrm{~g}$ of sodium monochloroacetate. The slurry was then filtered and the residue was suspended in $300 \mathrm{~mL}$ of methanol overnight. The next day, the slurry was neutralized with glacial acetic acid, followed by filtration and washing with $150 \mathrm{~mL}$ ethanol to remove undesirable residue. The final product (CMSP) was dried at $60{ }^{\circ} \mathrm{C}$ to constant weight.

Preparation of CMSP/Chitosan-Immobilized Activated Sludge (CMSP/Ch-AS) Beads. The PNP-acclimated activated sludge obtained from the SBR was subjected to centrifugation at $3000 \mathrm{rpm}$ for $20 \mathrm{~min}$. The supernatant was discarded and the activated sludge was washed again with $0.9 \mathrm{w} / \mathrm{v} \% \mathrm{NaCl}$ for rehydration and subjected to centrifugation at $3000 \mathrm{rpm}$ for $20 \mathrm{~min}$. Subsequently, the activated sludge was resuspended in distilled water to reach the required concentration for immobilization.

The optimization of immobilization process was carried out using one-factor experiment. The lower and upper limits of each factor were determined based on preliminary studies (data not shown), in which (1) the final solution produced is not too viscous to be extruded out from the needle attached to a syringe, and (2) the hydrogel formed is not of tear-shape. On this basis, the conditions decided for the immobilization of PNP-acclimated activated sludge were: CMSP concentration (6-10 wt \%), $\mathrm{Fe}^{3+}$ concentration $(0.05-0.4 \mathrm{M})$, crosslinking time (15-60 min), activated sludge concentration (5-10 $\left.\mathrm{g} \mathrm{L}^{-1}\right)$, and bead size $(3-5 \mathrm{~mm})$. Figure 1 shows the schematic diagram of the experimental setup for the immobilization process of activated sludge into CMSP/Ch hydrogel beads. The CMSP was dissolved in deionized water and its $\mathrm{pH}$ was adjusted to $\mathrm{pH} 7.4 \pm 0.2$, followed by the addition of predetermined concentration of

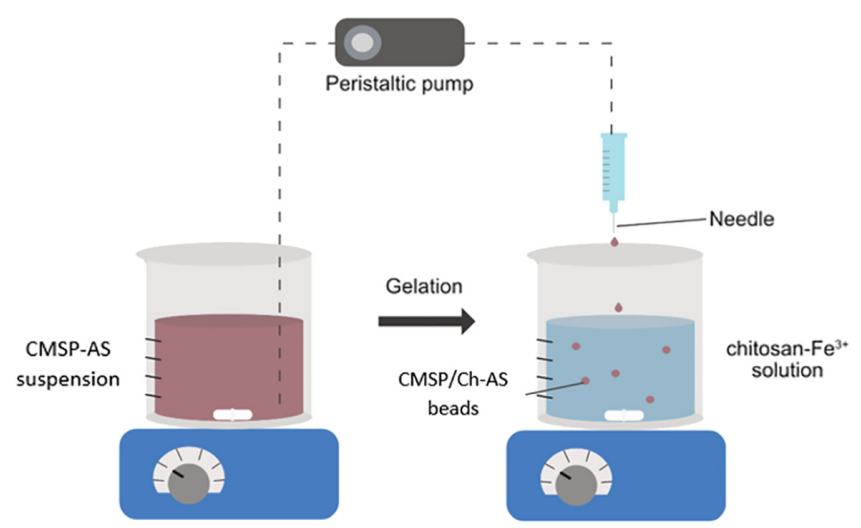

Figure 1. The schematic diagram of the experimental set-up for the formation of CMSP/Ch-AS beads. [Color figure can be viewed at wileyonlinelibrary.com] 


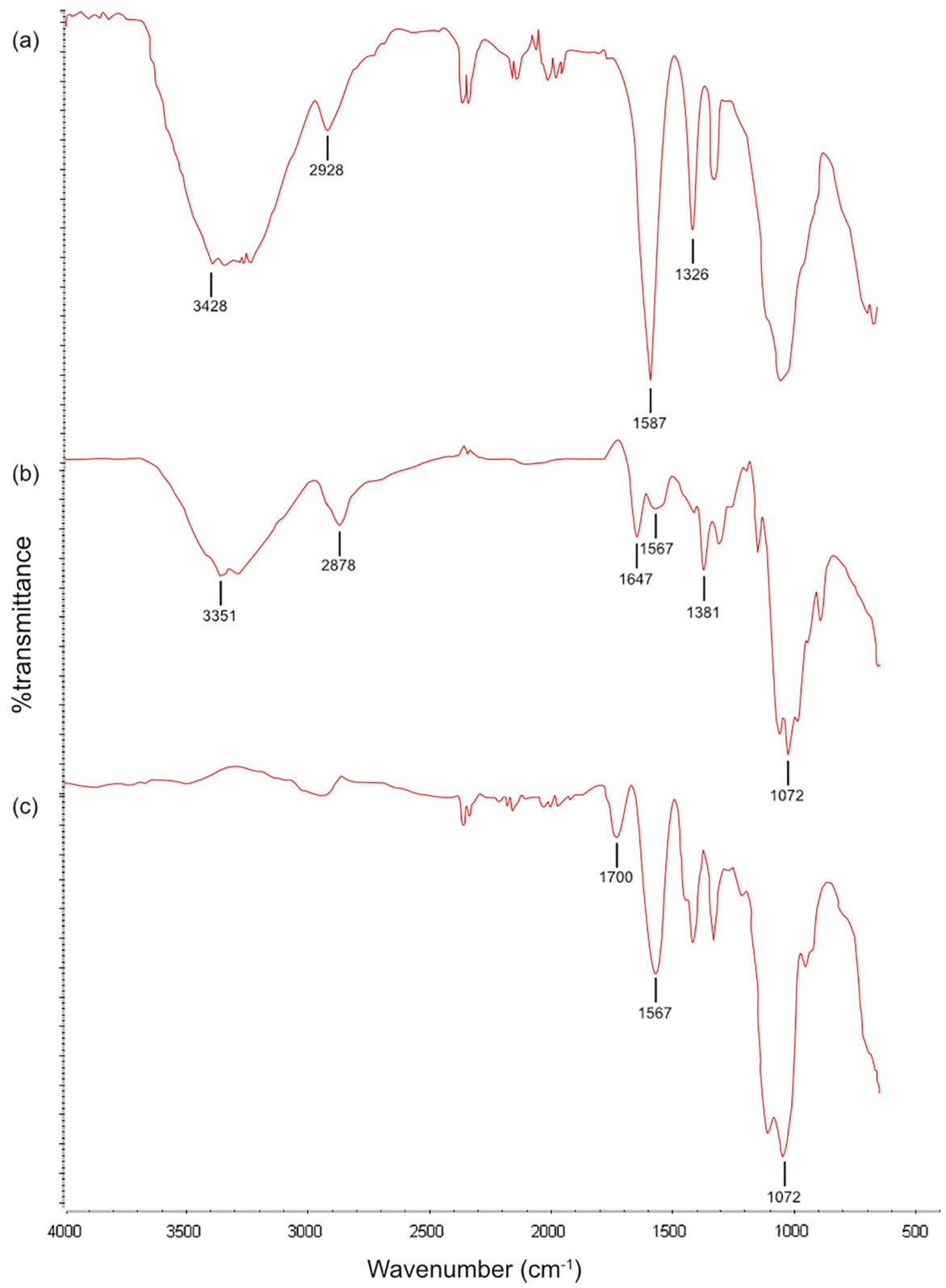

Figure 2. The FTIR spectra of (a) CMSP, (b) chitosan, and (c) CMSP/Ch hydrogel bead. [Color figure can be viewed at wileyonlinelibrary.com]

activated sludge. Subsequently, the 50-mL CMSP-AS mixture was mixed with $3 \mathrm{~mL}$ of $3 \%$ glutaraldehyde and then added dropwise using a needle attached to a $3-\mathrm{mL}$ syringe into a solution containing $50 \mathrm{~mL} \mathrm{Fe}{ }^{3+}$ and $50 \mathrm{~mL}$ of $0.4 \mathrm{w} / \mathrm{v} \%$ chitosan (with molecular weight of $600 \mathrm{kDa}$ ). The mixture was stirred for a predetermined time for crosslinking. The bead size was governed by the needle size. After CMSP/Ch-AS beads formation was completed, the mixture was filtered and the collected beads were washed using $0.01 \mathrm{M}$ phosphate buffer once and followed by distilled water for several times. The beads were temporarily stored at $4{ }^{\circ} \mathrm{C}$ in distilled water for not more than 1 day.

The optimized variables were determined based on the shortest duration in achieving complete biodegradation of PNP. The CMSP/Ch-AS beads prepared under different conditions were introduced into reaction flasks containing $25 \mathrm{mg} \mathrm{L}^{-1}$ PNP and nutrients. The time course of residual concentration of PNP in the bulk solution was monitored until PNP was completely degraded.

\section{Characterization}

The beads sizes were measured using image analyzer software (SigmaScan Pro 5, SPSS Inc.). The diameters of 30 beads were determined and expressed in terms of mean.

The functional groups of CMSP, chitosan, and CMSP/Ch hydrogel bead were characterized with a Fourier transform infrared (FTIR) spectrophotometer (Varian 640-IR, Joannesburg, South Africa) in the range of $4000-500 \mathrm{~cm}^{-1}$. The samples were oven-dried overnight to remove excess moisture before being ground using a mortar and pestle.

The morphology of activated sludge was analyzed using a scanning electron microscope (S3400N, Hitachi, Tokyo, Japan). About $1 \mathrm{~mL}$ of activated sludge was air dried. The sample was 

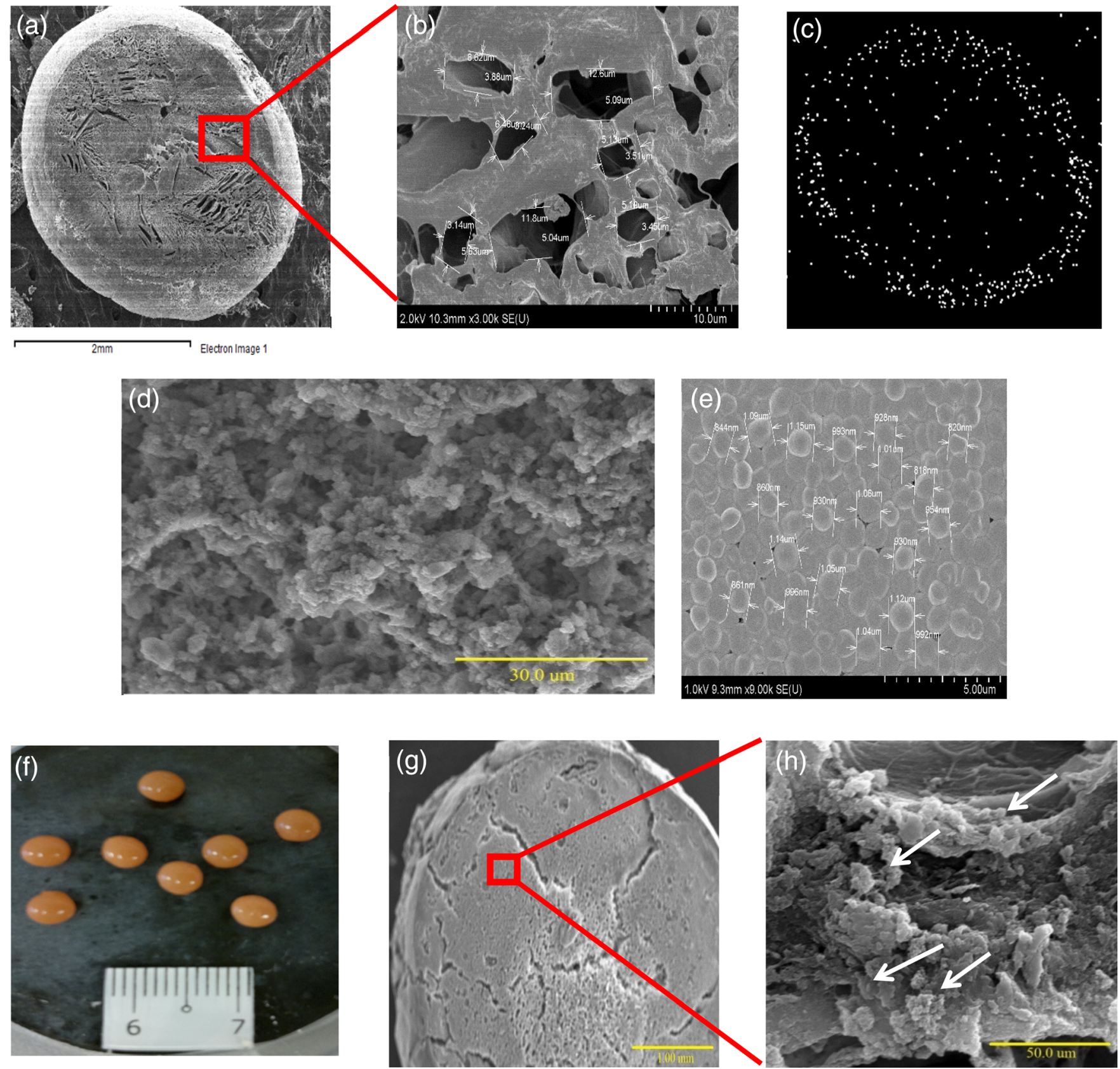

Figure 3. FESEM images of (a) surface of CMSP/Ch hydrogel bead and (b) cross section of CMSP/Ch hydrogel bead; (c) nitrogen element mapping for crosslinked CMSP/Ch hydrogel bead; SEM images of (d) and (e) suspended PNP-acclimated activated sludge; (f) image of the $3.94 \pm 0.04 \mathrm{~mm}$-CMSP/ChAS beads; FESEM images of ( $\mathrm{g}$ ) and (h) CMSP/Ch-AS bead after PNP biodegradation. [Color figure can be viewed at wileyonlinelibrary.com]

then fixed with $3 \%$ glutaraldehyde for $30 \mathrm{~min}$ and rinsed with a phosphate buffer solution. The fixed sample was frozen using liquid nitrogen and subjected to $16-\mathrm{h}$ freeze drying. Finally, the sample was sputter-coated with platinum prior to SEM observation.

The surface and cross section of the empty CMSP/Ch hydrogel bead and CMSP/Ch-AS bead were examined using a field emission scanning electron microscope (SU-8010, Hitachi, Tokyo, Japan) equipped with an energy-dispersive X-ray (Oxford-Horiba Inca XMax50, High Wycombe, England) at the acceleration voltage of $20 \mathrm{kV}$. The beads were sectioned to produce $1.0 \mathrm{~mm}$ thick pieces. Both intact and cross-sectioned beads were frozen at $-80{ }^{\circ} \mathrm{C}$ for $1 \mathrm{~h}$ and freeze-dried subsequently for $6 \mathrm{~h}$.

\section{Comparative Biodegradation of PNP by Suspended and Immobilized Activated Sludge}

The study of biodegradation of PNP at different initial concentration was carried out in batch mode. A total of $250 \mathrm{~mL}$ mixture containing PNP (200 or $400 \mathrm{mg} \mathrm{L}^{-1}$ ) and nutrients (composition as described in the previous section of Culturing of PNPAcclimated Activated Sludge) was prepared and CMSP/Ch-AS beads (consisted of $400 \mathrm{mg}$ of dried weight of PNP-acclimated 
activated sludge) were introduced into the reaction flask. The sampling was conducted at appropriate time intervals for the determination of residual PNP in the bulk solution until the attainment of complete biodegradation. The PNP concentration was determined spectrophotometrically using UV-Vis spectrophotometer (Shimadzu UV-1800, Kyoto, Japan) at $\lambda_{\max }$ of $400 \mathrm{~nm}^{22}$ At the end of biodegradation, chemical oxygen demand (COD) was determined using a COD Vario Photometer (Tintometer, GmbH, Germany) to ascertain the attainment of mineralization. For comparison, the experiment was repeated using suspended activated sludge at the same dried weight. From the preliminary experiments (results not shown), the PNP removal via vitalization and adsorption was negligible. All the experiments were carried out three times and the mean values were reported.

Reusability of CMSP/Ch-Immobilized Activated Sludge Beads The investigation on the reusability of the CMSP/Ch-AS beads was conducted using different initial concentration of PNP at 200 and $400 \mathrm{mg} \mathrm{L}^{-1}$, respectively. The biodegradation of PNP was repeated using the same batch of CMSP/Ch-AS beads until noticeable disintegration of beads or leaching of activated sludge from the beads was observed. At the end of each biodegradation cycle, the CMSP/Ch-AS beads were obtained by filtration and washed with distilled water before they were used for the next cycle of biodegradation.

\section{Statistical Analyses}

Statistical analyses were performed using SPSS version 21. All results are expressed in mean values. Parameters were compared using one-way analysis of variance (ANOVA) with subgroup analyses using pairwise multiple comparison procedures (Tukey test) for multiple testing. The $p$-value of $<0.05$ was considered as statistically significant. All assays were conducted in at least triplicate and repeated in three separate experiments.

\section{RESULTS AND DISCUSSION}

\section{Characterization}

FTIR Analyses. Figure 2 shows the FTIR spectra for CMSP, chitosan, and CMSP/Ch hydrogel beads crosslinked with ferric ion. The characteristic peaks (in $\mathrm{cm}^{-1}$ ) obtained for raw CMSP are: 3428 (stretching vibrations of $-\mathrm{OH}$ groups), 2928 (stretching vibration of $\mathrm{C}-\mathrm{H}$ ), 1587 (stretching vibration of $\mathrm{COO}^{-}$), and 1326 (bending vibration of $-\mathrm{OH}){ }^{23}$ Whereas for chitosan, the peaks obtained are: 3551 (stretching vibrations of the $-\mathrm{OH}$ and $-\mathrm{NH}$ groups), 2878 ( $\mathrm{C}-\mathrm{H}$ stretching), 1647 (amide II band $\mathrm{N}-\mathrm{H}$ bending and $\mathrm{C}=\mathrm{O}$ carbonyl stretching of acetyl group), 1567 (stretching vibration of amine group), 1381 ( $\mathrm{C}-\mathrm{N}$ stretching or $\mathrm{O}-\mathrm{H}$ bonding), and 1072 (alcoholic $\mathrm{C}-\mathrm{O}$ and $\mathrm{C}-\mathrm{N}$ stretching). ${ }^{24}$

After crosslinking with ferric ion, the carbonyl group was found to shift to a higher wavenumber, from about 1647 (spectrum 2 (b)) to $1700 \mathrm{~cm}^{-1}$ (spectrum 2(c)). Comparing spectra 2(a) and (c), the intensity reductions observed for peaks at 3428 and $1587 \mathrm{~cm}^{-1}$, implied that the hydroxyl and carboxyl groups were involved in the binding of ferric through coordination and electrostatic interactions. ${ }^{25}$ The existence of $1567 \mathrm{~cm}^{-1}$ peak signified the formation of polyelectrolyte complex due to the
Table I. The Percentages of Elements in Empty CMSP/Ch Hydrogel Beads under the EDX Analyses

\begin{tabular}{llc}
\hline Surface & Element & Weight \% \\
\hline Inner & C & 39.68 \\
& O & 15.07 \\
& Fe & 45.25 \\
Outer & C & 46.54 \\
& O & 35.63 \\
& Si & 4.76 \\
& Fe & 13.07 \\
\hline
\end{tabular}

electrostatic interaction between the carboxyl of CMSP and amine of chitosan. ${ }^{26}$

\section{Morphology and Elemental Studies}

Figure 3(a) shows the porous and smooth surface of a $\mathrm{Fe}$ crosslinked empty CMSP/Ch hydrogel bead. The cross section of a $\mathrm{CMSP} / \mathrm{Ch}$ hydrogel bead is shown in Figure 3(b), and randomsized and irregular shapes of pores can be seen. Based on the preliminary study (data not shown), the formation of stable hydrogel beads by crosslinking CMSP of DS 0.8 with ferric ions was unsuccessful in the absence of chitosan. The stabilization of the architecture of the beads was achieved with the addition of chitosan during the gelation process. Figure 3(c) shows the FESEM-EDX nitrogen element mapping in a CMSP/Ch hydrogel bead that reveals the distribution of nitrogen (which was originated from chitosan) in the bead. Owing to the high molecular weight of the chitosan used, it was observed that the chitosan mainly reacted with the CMSP at the outer most of the bead, forming a layer of coating on the bead surface. The role of chitosan in improving the stability was also reported by Lee et al., ${ }^{21}$ in which high-molecularweight chitosan formed a coating on the alginate microparticles.

Figure 3(d) shows the surface morphology of suspended PNPacclimated activated sludge. The aggregation of sludge cells and extracellular polymeric substances was observed to form clump of rough surface. The average size of the activated sludge was found to be $979.35 \pm 105.18 \mathrm{~nm}$ [Figure 3(e)]. Figure 3(f) shows the image of 4 -mm-CMSP/Ch-AS beads. The cross section of CMSP/Ch-AS bead after one cycle of PNP biodegradation process is presented in Figure 3(g,h). An observable gradient of pore size was detected with larger pore size exhibited at the center of the beads compared to the outer section [Figure $3(\mathrm{~g})]$. The smaller pores at the outer section could be plausibly explained by the presence of immobilized activated sludge which had covered most of the pores. Also, the entrapped activated sludge can be observed from the denser or darker surface compared to the center of beads where activated sludge was relatively lesser. The clumps of sludge which can be seen in Figure 3(h) (see arrow) were found absent in empty CMSP/Ch hydrogel bead [Figure 3 (b)], indicating that the activated sludge was successfully immobilized forming CMSP/Ch-AS beads.

The FESEM-EDX analyses (Table I) revealed that the inner and outer of the empty beads contain iron. The inner section of the beads has a higher amount of iron compared to the outer layer. 
This shows that the iron has been successfully crosslinked into the matrices of CMSP/Ch hydrogel beads.

\section{Optimization of Immobilization of PNP-Acclimated Activated Sludge}

The effects of concentrations of CMSP, PNP-acclimated activated sludge, crosslinker as well as crosslinking time and bead size on the biodegradation time of PNP are presented in Figure 4. For the variable of CMSP concentration, the shortest duration to achieve complete biodegradation was observed for 6 and 7 w/v\% CMSP. At higher concentration of CMSP, the formation of extensive bonding was promoted resulting in the more compact matrix structure of beads. Consequently, diffusion of PNP and oxygen into the beads was limited ${ }^{5}$ and thus a longer time was required for complete PNP biodegradation. In this study, aggregation was observed when $6 \mathrm{w} / \mathrm{v} \%$ CMSP was used. Considering the statistically insignificant difference of performance for CMSP at 6 and $7 \mathrm{w} / \mathrm{v} \%, 7 \mathrm{w} / \mathrm{v} \%$ CMSP was chosen as the optimum CMSP concentration and used for the subsequent studies.

\section{(a)}

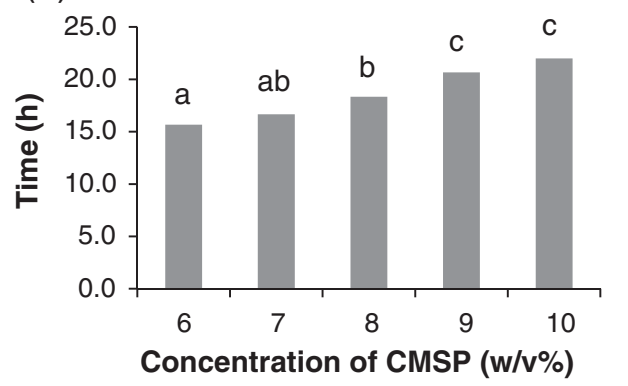

(c)

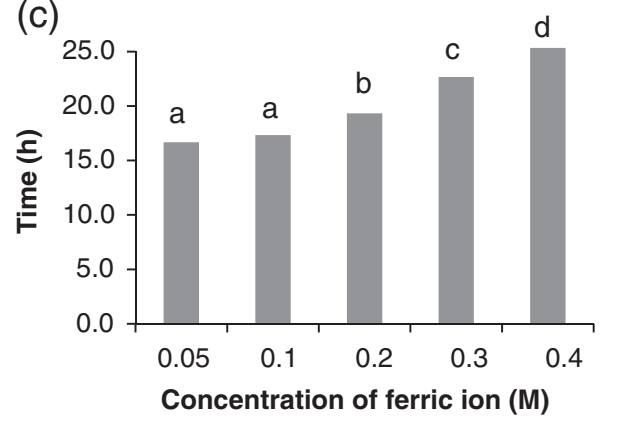

For the factor of concentration of activated sludge, the optimum concentration was found to be $9 \mathrm{~g} \mathrm{~L}^{-1}$ with complete PNP degradation achieved within $15.3 \mathrm{~h}$. It was perceived that the effect of the concentration of activated sludge on the biodegradation time in the immobilized system was relatively less notable in comparison to that of the suspended system. There was no significant decrease in the biodegradation time when the immobilized activated sludge concentration was increased from 6 to $10 \mathrm{~g} \mathrm{~L}^{-1}$. In contrast, in the study of Sam and $\mathrm{Ng}^{1}$ on the phenol degradation using suspended phenol-acclimated activated sludge, the complete degradation was reported to reduce from 9 to $1 \mathrm{~h}$ when the concentration activated sludge was increased by only $300 \mathrm{mg} \mathrm{L}^{-1}$, from 100 to $400 \mathrm{mg} \mathrm{L}^{-1}$. This phenomenon could be explained by the additional step of diffusion of substrates into the hydrogel beads and thus the limitation of the accessible substrates to the immobilized activated sludge.

Figure $4(\mathrm{c}, \mathrm{d})$ shows the increasing biodegradation time with increasing concentration of ferric ion and crosslinking time, respectively. Similar to the effect of CMSP concentration, a higher concentration of crosslinker and longer period of the

(b)

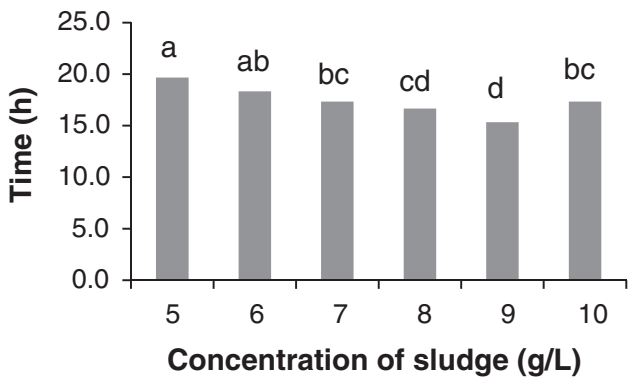

(d)

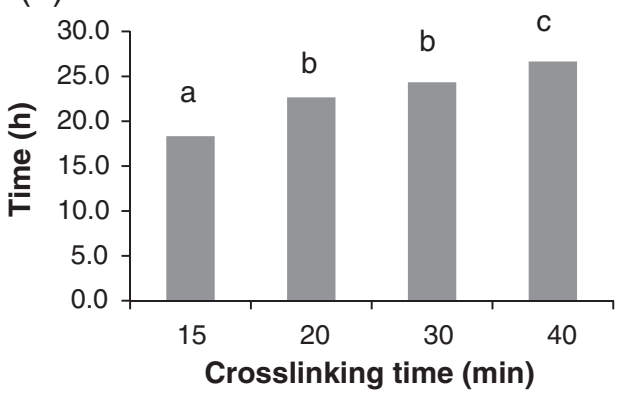

(e)

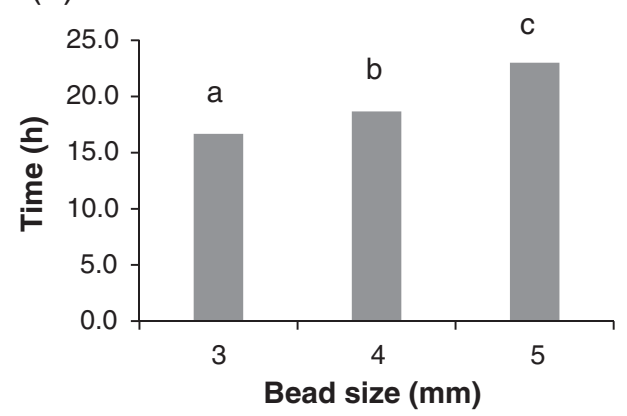

Figure 4. The effects of (a) CMSP concentration, (b) activated sludge concentration, (c) ferric ion concentration, (d) crosslinking time, and (e) bead size on the biodegradation time of $25 \mathrm{mg} \mathrm{L}^{-1}$ PNP. Values with the same letters are not significantly different (one-way ANOVA, Tukey test, $p<0.05$ ). 


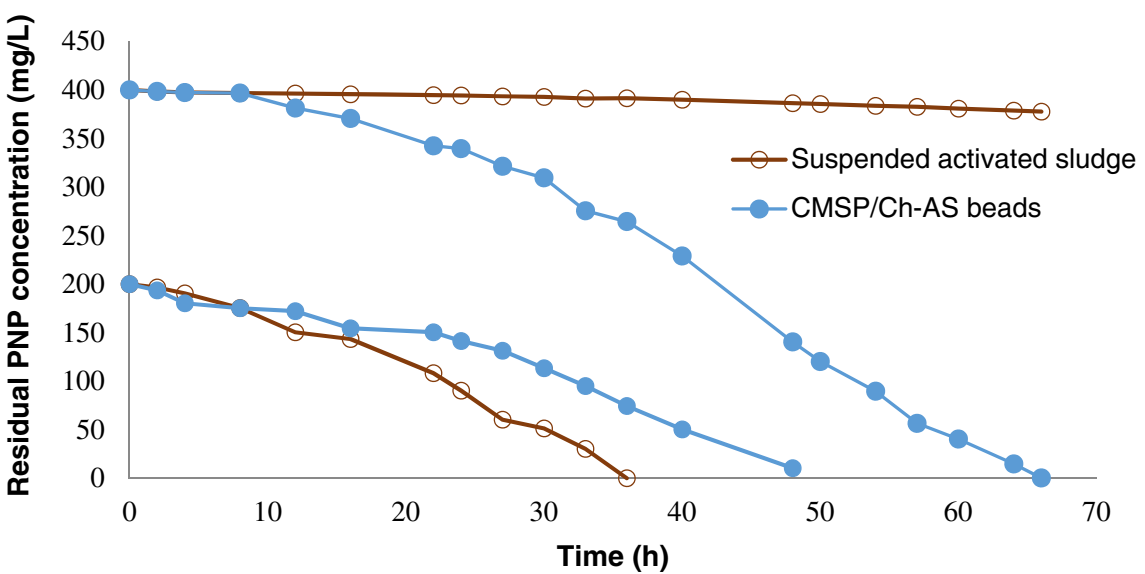

Figure 5. Time courses of residual concentration during biodegradation of PNP by CMSP/Ch-AS beads and suspended activated sludge at initial concentration of 200 and $400 \mathrm{mg} \mathrm{L}^{-1}$. [Color figure can be viewed at wileyonlinelibrary.com]

crosslinking process resulted in excessive crosslinking in the CMSP/Ch-AS beads and consequently less efficient diffusion of PNP and oxygen into the beads. CMSP/Ch-AS beads were found to be relatively more fragile when $0.05 \mathrm{M}$ of ferric ion was used, thus the optimum concentration was determined to be $0.1 \mathrm{M}$ ferric ion.

For the bead size, its influence could be associated with the surface area. The biodegradation time was observed to be shorter when smaller bead size was used. A similar trend was noted by Lee et al. ${ }^{6}$ treating phenol using immobilized activated sludge. Smaller bead size exhibited higher surface area and thus allowing more diffusion of PNP and oxygen into the beads. The incidence of diffusion restrictions when the bigger diameter of beads was used has also been reported by See et al. ${ }^{3}$ For the bead size range investigated in this study, beads with $3.0 \mathrm{~mm}$ in diameter were determined to be the optimum condition.

\section{Biodegradation of PNP by Suspended and Immobilized Activated Sludge}

The studies of biodegradation of PNP at different initial concentration of 200 and $400 \mathrm{mg} \mathrm{L}^{-1}$ were carried out using suspended and immobilized activated sludge (CMSP/Ch-AS beads), respectively, and the respective time courses of residual PNP in the bulk solution are exhibited in Figure 5. In this study, the activated sludge was acclimated to $200 \mathrm{mg} \mathrm{L}^{-1}$ of PNP. When the same PNP concentration was used in the biodegradation study, both immobilized and suspended activated sludge were able to completely degrade $\mathrm{PNP}$, in which mineralization was ascertained by the low COD concentration of $<50 \mathrm{mg} \mathrm{L}^{-1}$. Longer biodegradation time for CMSP/Ch-AS beads was attributed to the additional diffusion process prior to biodegradation.

Nonetheless, when the PNP concentration was doubled to $400 \mathrm{mg} \mathrm{L}^{-1}$, the toxicity effect of PNP on the biodegradation ability of suspended activated sludge was revealed by the nearly plateau PNP concentration within the experimental period (Figure 5). In contrast, CMSP/Ch-AS beads showed the capability of performing complete biodegradation of PNP within $66 \mathrm{~h}$. In comparison to the direct contact of suspended activated sludge to the high PNP concentration, the immobilized activated sludge was protected from the PNP toxicity due to the diffusion gradient of toxic PNP into the beads. Banerjee and Ghoshal ${ }^{12}$ reported a similar protection and therefore the improved phenol tolerance and more efficient phenol biodegradation by alginate-immobilized pure strains.

\section{Reusability of CMSP/Ch-AS Beads}

For reusability study, at the initial PNP concentration of $200 \mathrm{mg}$ $\mathrm{L}^{-1}$, it was found that the CMSP/Ch-AS beads could be reused for three consecutive cycles of biodegradation without significant deterioration in the biodegradation ability. PNP removal efficiency of more than $99 \%$ was achieved at each cycle. However, at the fourth cycle, CMSP/Ch-AS beads breakage was noticed. When the initial PNP concentration was increased to $400 \mathrm{mg} \mathrm{L}^{-1}$, the breakage was observed earlier, at the third cycle. The disintegration of beads was caused by the excessive growth of activated sludge within the beads under high supply of PNP. The effect of initial substrate concentration on the reusability of immobilized activated sludge beads was also reported by Toh et al. ${ }^{13} \mathrm{In}$ their study, the reusability of poly(vinyl alcohol) (PVA) hydrogelbiomass treating $o$-cresol was found to reduce from three to two cycles when the initial concentration of $o$-cresol was increased from 100 to $400 \mathrm{mg} \mathrm{L}^{-1}$. From the reusability test, it can be concluded that the CMSP/Ch hydrogel beads were feasible to be utilized as the immobilization medium for activated sludge and the biodegradation performance was comparable to that of conventional widely used polymeric material such as PVA.

\section{CONCLUSIONS}

In the study, CMSP extracted from sago waste was successfully used to immobilize PNP-acclimated activated sludge in the presence of chitosan forming CMSP/Ch-AS beads. The chitosan coating on the surface of the beads resulted in the enhanced stability of the beads. In comparison to suspended activated sludge, the CMSP/Ch-immobilized activated sludge was protected by the polymeric matrix and the CMSP/Ch-AS beads were able to mineralize PNP at the initial concentration of $400 \mathrm{mg} \mathrm{L}^{-1}$, a concentration that was double of the acclimation concentration. The optimized conditions in preparing CMSP/Ch-AS beads for biodegradation of $25 \mathrm{mg} \mathrm{L}^{-1} \mathrm{PNP}$ were found to be $7 \mathrm{w} / \mathrm{v} \%$ CMSP, $9 \mathrm{~g} \mathrm{~L}^{-1}$ of activated sludge, and $0.1 \mathrm{M}$ ferric ion with $15 \mathrm{~min}$ of crosslinking time. The 
reusability of CMSP/Ch-AS beads was observed to depend on the initial concentration of PNP, reaching three and two cycles of usage, respectively, in biodegrading 200 and $400 \mathrm{mg} \mathrm{L}^{-1} \mathrm{PNP}$.

\section{ACKNOWLEDGMENTS}

The work was supported by Monash University Malaysia and Universiti Sains Malaysia (USM) (304.PKIMIA.6316242). One of the authors, Suat Peng Sam, would like to acknowledge the financial support from USM fellowship scheme.

\section{REFERENCES}

1. Sam, S. P.; Ng, S. L. J. Phys. Sci. 2017, 28, 53.

2. Gemini, V. L.; Gallego, A.; de Oliveira, V. M.; Gomez, C. E.; Manfio, G. P.; Korol, S. E. Int. Biodeterior. Biodegrad. 2005, 55, 103.

3. See, S.; Lim, J. W.; Lim, P. E.; Seng, C. E.; Ng, S. L.; Adnan, R. Desalin. Water Treat. 2014, 52, 7951.

4. Alves, A. P. A.; Lima, P. S.; Dezotti, M.; Bassin, J. P. Int. Biodeterior. Biodegrad. 2017, 123, 146.

5. An, M.; Lo, K. V. J. Environ. Sci. Health, Part A. 2001, 36, 101.

6. Lee, S.-Y.; Chun, Y.-N.; Kim, S.-I. J. Ind. Eng. Chem. 2009, 15, 323.

7. Zhang, L.-S.; Wu, W.-Z.; Wang, J.-L. J. Environ. Sci. 2007, 19, 1293.

8. Dzionek, A.; Wojciezyńska, D.; Guzik, E. Electron. J. Biotechnol. 2016, 23, 28.

9. Wang, J. L.; Hou, W. H.; Qian, Y. Biotechnol. Tech. 1995, 10, 203.

10. Wang, J. L.; Qian, Y. Chemosphere. 1999, 38, 3109.

11. Qiao, X.; Liu, Z.; Liu, X.; Zeng, Y.; Zhang, Z. Process Biochem. 2010, 45, 1342.
12. Banerjee, A.; Ghoshal, A. K. Int. Biodeterior. Biodegrad. 2011, 65, 1052.

13. Toh, R. H.; Seng, C. E.; Lim, P. E.; Adnan, R.; Sujar, A. N. A. Desalin. Water Treat. 2013, 51, 7216.

14. Leong, K.-Y.; Adnan, R.; Lim, P.-E.; Ng, S.-L.; Seng, C.-E. Environ. Sci. Pollut. Res. 2017, 24, 20959.

15. Dahlan, N. A.; Ng, S. L.; Pushpamalar, J. J. Appl. Polym. Sci. 2017, 134, 44271.

16. Lai, J. C.; Rahman, W. A.; Toh, W. Y. Ind. Crops Prod. 2013, 45, 319.

17. Tan, H. L.; Wong, Y. Y.; Myniyandy, S.; Hashim, K.; Pushpamalar, J. J. Appl. Polym. Sci. 2016, 133, 43625.

18. Thenapakiam, S.; Kumar, D. G.; Pushpamalar, J.; Saravanan, M. Carbohydr. Polym. 2013, 94, 356.

19. Lam, Y. L.; Muniyandy, S.; Kamaruddin, H.; Mansor, A.; Janarthanan, P. Radiat. Phys. Chem. 2015, 106, 213.

20. Saravanan, M.; Thenapakiam, S.; Anand, K. V.; Pushpamalar, J. Polymers. 2015, 7, 1099.

21. Lee, J.; Cha, D.; Park, H. J. Agric. Food Chem. 2004, 5, 7300 .

22. Xing, X. H.; Inoue, T.; Tanji, Y.; Unno, H. J. Biosci. Bioeng. 1999, 87, 372.

23. Pushpamalar, V.; Langford, S.; Ahmad, M.; Lim, Y. Y. Carbohydr. Polym. 2006, 64, 312.

24. Pereira, F. A.; Sousa, K. S.; Cavalcanti, G. R.; Fonseca, M. G.; de Souza, A. G.; Alves, A. P. Int. J. Biol. Macromol. 2013, 61, 471.

25. Lv, L.; Xie, Y.; Liu, G.; Liu, G.; Yu, J. J. Environ. Sci. 2014, 26, 792.

26. Rosca, C.; Popa, M. I.; Lisa, G.; Chitanu, G. C. Carbohydr. Polym. 2005, 62, 35. 